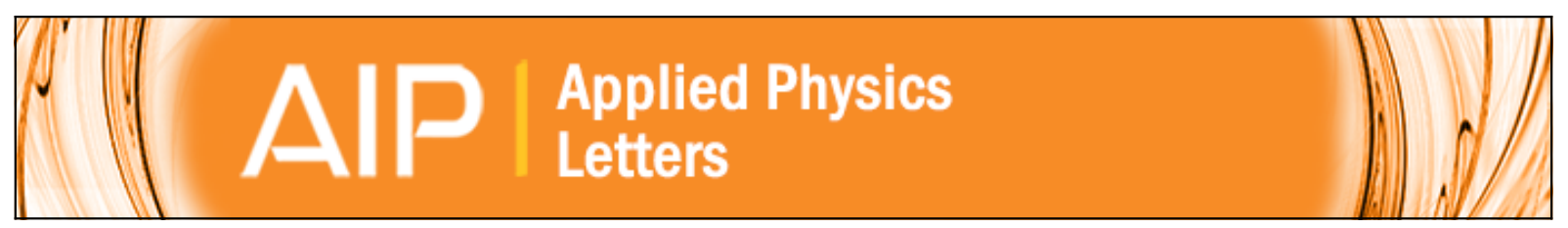

\title{
Negative differential resistance in direct bandgap GeSn p-i-n structures
}

C. Schulte-Braucks, D. Stange, N. von den Driesch, S. Blaeser, Z. Ikonic, J. M. Hartmann, S. Mantl, and D. Buca

Citation: Applied Physics Letters 107, 042101 (2015); doi: 10.1063/1.4927622

View online: http://dx.doi.org/10.1063/1.4927622

View Table of Contents: http://scitation.aip.org/content/aip/journal/apl/107/4?ver=pdfcov

Published by the AIP Publishing

\section{Articles you may be interested in}

Electroluminescence from GeSn heterostructure pin diodes at the indirect to direct transition

Appl. Phys. Lett. 106, 091103 (2015); 10.1063/1.4913688

GeSn p-i-n waveguide photodetectors on silicon substrates

Appl. Phys. Lett. 105, 231109 (2014); 10.1063/1.4903881

Compressively strained SiGe band-to-band tunneling model calibration based on p-i-n diodes and prospect of strained SiGe tunneling field-effect transistors

J. Appl. Phys. 116, 214506 (2014); 10.1063/1.4903288

Competition of optical transitions between direct and indirect bandgaps in Ge1-xSnx

Appl. Phys. Lett. 105, 051104 (2014); 10.1063/1.4892302

Ge-Sn semiconductors for band-gap and lattice engineering

Appl. Phys. Lett. 81, 2992 (2002); 10.1063/1.1515133

Frustrated by

old technology?

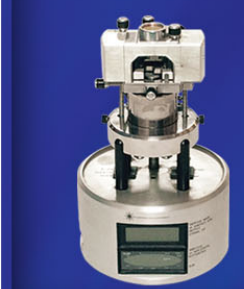

Is your AFM dead

and can't be repaired?

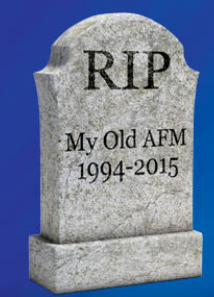

Sick of bad customer support?

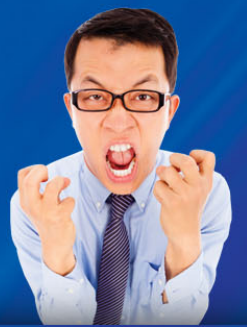

It is time to upgrade your AFM

Minimum $\$ 20,000$ trade-in discount for purchases before August 31st

Asylum Research is today's technology leader in AFM

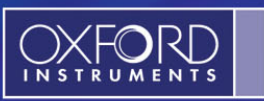




\title{
Negative differential resistance in direct bandgap GeSn p-i-n structures
}

\author{
C. Schulte-Braucks, ${ }^{1}$ D. Stange, ${ }^{1}$ N. von den Driesch, ${ }^{1}$ S. Blaeser, ${ }^{1}$ Z. Ikonic, ${ }^{2}$ \\ J. M. Hartmann, ${ }^{3}$ S. Mantl, ${ }^{1}$ and D. Buca ${ }^{1}$ \\ ${ }^{1}$ Peter Grünberg Institut (PGI-9) and JARA-FIT, Forschungszentrum Jülich GmbH, 52428 Jülich, Germany \\ ${ }^{2}$ Institute of Microwaves and Photonics, School of Electronic and Electrical Engineering, \\ University of Leeds, Leeds LS2 9JT, United Kingdom \\ ${ }^{3}$ University of Grenoble Alpes, F-38000 Grenoble, France and CEA, LETI, MINATEC Campus, \\ F-38054 Grenoble, France
}

(Received 24 May 2015; accepted 15 July 2015; published online 28 July 2015)

\begin{abstract}
Certain GeSn alloys are group IV direct bandgap semiconductors with prospects for electrical and optoelectronical applications. In this letter, we report on the temperature dependence of the electrical characteristics of high Sn-content $\mathrm{Ge}_{0.89} \mathrm{Sn}_{0.11} p$ - $i$ - $n$ diodes. NiGeSn contacts were used to minimize the access resistance and ensure compatibility with silicon technology. The major emphasis is placed on the negative differential resistance in which peak to valley current ratios up to 2.3 were obtained. TCAD simulations were performed to identify the origin of the various current contributions, providing evidence for direct band to band tunneling and trap assisted tunneling. (C) 2015 AIP Publishing LLC. [http://dx.doi.org/10.1063/1.4927622]
\end{abstract}

The recent experimental proof of GeSn as group IV direct bandgap semiconductor ${ }^{1}$ will stimulate novel applications in photonics and electronics. Due to its low direct bandgap energy and silicon compatibility, GeSn alloys are particularly interesting for tunneling field-effect transistors (TFETs) for ultra-low power electronics. ${ }^{2,3} p-i-n$ diodes are the basic building blocks of tunneling-FETs enabling band-to-band tunneling (BTBT) allowing the extraction of material parameters such as tunneling masses. ${ }^{4}$ Since the first demonstration of a tunneling diode by Esaki, ${ }^{5}$ showing the characteristic negative differential resistance (NDR), tunneling diodes have been fabricated with different indirect group IV semiconductors such as $\mathrm{Si}^{6}{ }^{6} \mathrm{Ge},{ }^{7}$ and $\mathrm{SiGe} .{ }^{8}$ However, the highest tunneling current densities were achieved on direct, low bandgap III-V semiconductors like InAs ${ }^{9}$ adding sustained effort for integrating III-V semiconductors on $\mathrm{Si}$ wafers. Recently developed GeSn alloys with Sn contents above $10 \%$ provide new perspectives for Si electronics by combining the advantages of III-V compounds as low effective mass of $\Gamma$-electrons and the direct bandgap with stateof-the-art Si platform technology. Several papers on GeSn MOSFETs $^{10,11}$ and even TFETs ${ }^{2,12}$ have demonstrated that GeSn alloys can be technologically integrated. However, these devices had low Sn-contents below the indirect to direct transition.

In this letter, we present the fabrication and electrical characterization of direct bandgap group IV $p-i$ - $n$ diodes with emphasis on BTBT. First, we address intrinsic material properties, as bandgap and effective masses, obtained by calculations followed by the electrical characterization of Esaki tunneling diodes. Finally, the experimental results are compared with device simulations.

GeSn alloys pseudomorphically grown on Ge are under high biaxial compressive strain and thus are indirect semiconductors even with $\mathrm{Sn}$ contents of $10 \% .{ }^{13}$ By reducing the compressive strain, e.g., by growing thicker layers, the $\Gamma$-valley decreases faster in energy than the L-valley and GeSn becomes a direct semiconductor. ${ }^{13}$ Fig. 1(a) depicts the calculated bandgap vs. compressive strain, as obtained by the $8 \times 8 \mathrm{k} \cdot \mathrm{p}$ method including strain, ${ }^{14}$ pointing out the necessity of strain relaxation for achieving direct bandgap GeSn. The strain at which this transition takes place depends on the $\mathrm{Sn}$ content and for $\mathrm{Ge}_{0.89} \mathrm{Sn}_{0.11}$, relevant in this work, is about $-0.8 \%$ (Fig. 1(a)). In addition to the advantage of a direct bandgap, GeSn layers offer also smaller effective masses for both holes and electrons ${ }^{15}$ suggesting themselves for tunneling based devices as TFETs. ${ }^{15}$ The heavy hole $(\mathrm{HH})$, light hole $(\mathrm{LH})$, and the $\Gamma$-valley electron masses in-plane [100] and out of plane [001] direction as calculated with the $8 \times 8 \mathrm{k} \cdot \mathrm{p}$ method including strain $^{14}$ are plotted in Figs. 1(b) and 1(c) for a set of Sn contents. The HH mass in tunneling direction [001] is independent of strain and decreases only slightly from $0.221 \mathrm{~m}_{0}$ to $0.219 \mathrm{~m}_{0}$ when the $\mathrm{Sn}$-content changes from $9 \%$ to $12 \%$, respectively. Here, strain relaxation is beneficial as well enabling small tunneling masses and consequently high BTBT-rates. Independent of the directness $\left(\mathrm{E}_{\mathrm{L}}-\mathrm{E}_{\Gamma}\right)$ of the GeSn alloys, compressive strain splits the valence band such that the HH becomes top in energy. The $\Gamma-\mathrm{HH}$ and $\Gamma$-LH transitions are both direct tunneling processes without any change of the in-plane wave-vector $\mathrm{k}_{\|}$. However, due to $\mathrm{p}_{\mathrm{x}, \mathrm{y}}$-symmetry of $\mathrm{HH}$ wave-functions and s-symmetry of conduction bands wave-functions, the tunneling is not possible at $\mathrm{k}_{\|}=0$, but it sets in as $\mathrm{k}_{\|}$increases, due to band-mixing. The $\Gamma$ - $\mathrm{LH}$ tunneling is possible even for $\mathrm{k}_{\|}=0$. Important factors influencing the tunneling current are $\Gamma$ and $L$ densities of states (DOS) defining the population of the electronic states. This is shown in Fig. 1(d) as a function of temperature for different doping levels. The $\Gamma$-electron density increases with decreasing temperature, as electrons condensate into the lowest energy levels, but their fraction becomes lower with increasing doping: it is $100 \%$ for a total electron density of $1 \times 10^{17} \mathrm{~cm}^{-3}$ but it decreases to just $32 \%$ for $10^{18} \mathrm{~cm}^{-3}$ total electron density. The remaining electrons reside in the L-valley. Table I summarizes the material parameters at $300 \mathrm{~K}$ for the alloys 

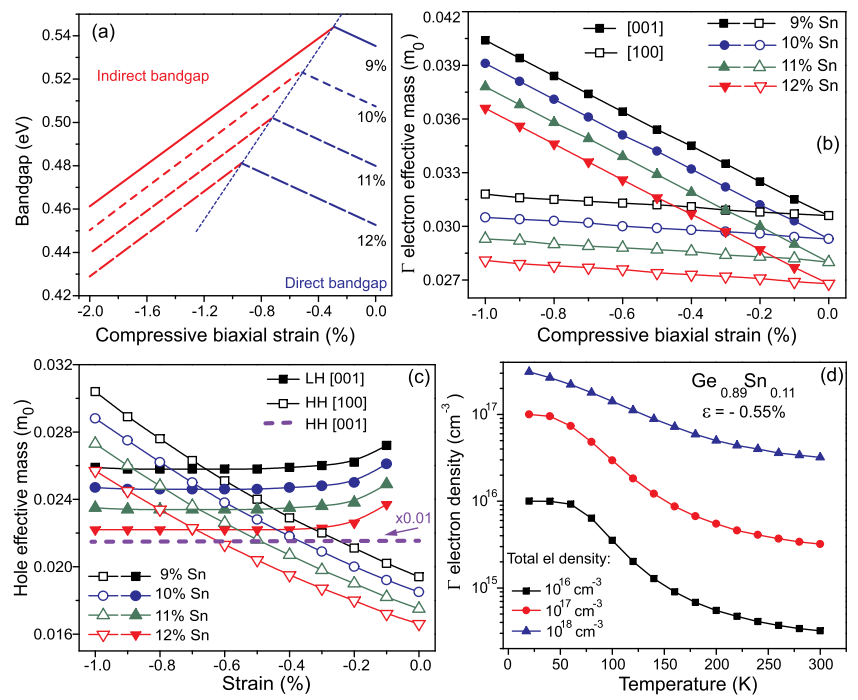

FIG. 1. Calculated (a) bandgap, (b) $\Gamma$-electron effective mass, and (c) lowest hole effective masses for GeSn alloys with Sn contents between 9\% and $12 \%$ as function of biaxial compressive strain. (d) $\Gamma$-valley population for $\mathrm{Ge}_{0.89} \mathrm{Sn}_{0.11}$ under compressive strain of $-0.55 \%$ for different $\mathrm{n}$-type doping.

discussed in this letter. These values are later used in TCAD simulations of the tunneling diodes.

Epitaxial growth of the GeSn layer stack was performed in an industry-compatible $200 \mathrm{~mm}$ AIXTRON TRICENT ${ }^{\circledR}$ reduced pressure chemical vapor deposition (CVD) reactor. Precursors as $\mathrm{Ge}_{2} \mathrm{H}_{6}$ and $\mathrm{SnCl}_{4}$ enable the growth of $\mathrm{GeSn}$ alloys at temperatures of about $350^{\circ} \mathrm{C}$ without undesired $\mathrm{Sn}$ segregation and surface precipitations. ${ }^{3,16}$ For a high quality of the GeSn alloys and an easier device fabrication, the complete $p-i$-n layer stack was grown on a $2.5 \mu \mathrm{m}$ thick highly $\mathrm{p}^{+}$-doped ${ }^{17}\left(\mathrm{~B}, 2 \times 10^{19} \mathrm{~cm}^{-3}\right) \mathrm{Ge}$ virtual substrate (Ge-VS). The latter was grown on $\mathrm{Si}(001)$ using $\mathrm{GeH}_{4}$ and $\mathrm{B}_{2} \mathrm{H}_{6} \cdot{ }^{17}$ In order to avoid implantation induced damage, in-situ doped $\mathrm{p}^{+}$and $\mathrm{n}^{+}$type doped GeSn layers were epitaxially grown using $\mathrm{B}_{2} \mathrm{H}_{6}$ and $\mathrm{PH}_{3}$, respectively. The electrically active carrier concentrations in the doped GeSn layers were about $2 \times 10^{19} \mathrm{~cm}^{-3}$ in the bottom $50 \mathrm{~nm} \mathrm{GeSn} \mathrm{layer}$ (B, p-type) and $1 \times 10^{20} \mathrm{~cm}^{-3}(\mathrm{P}, \mathrm{n}$-type) in the top $80 \mathrm{~nm}$ GeSn alloy as determined by electrochemical capacitancevoltage (ECV) profiling (see Fig. 4(a)). The scope of this letter is to show band-to band tunneling in a direct bandgap alloy. To that end, the GeSn layers are grown thicker than the critical thickness for plastic relaxation in order to promote strain relaxation and thereby realize an indirect to direct bandgap transition. The residual compressive strain in the partially relaxed $\mathrm{Ge}_{0.89} \mathrm{Sn}_{0.11}$ alloy is $-0.55 \%$. Snconcentrations and strain values have been verified using Rutherford-backscattering-spectroscopy and x-ray diffraction, respectively.

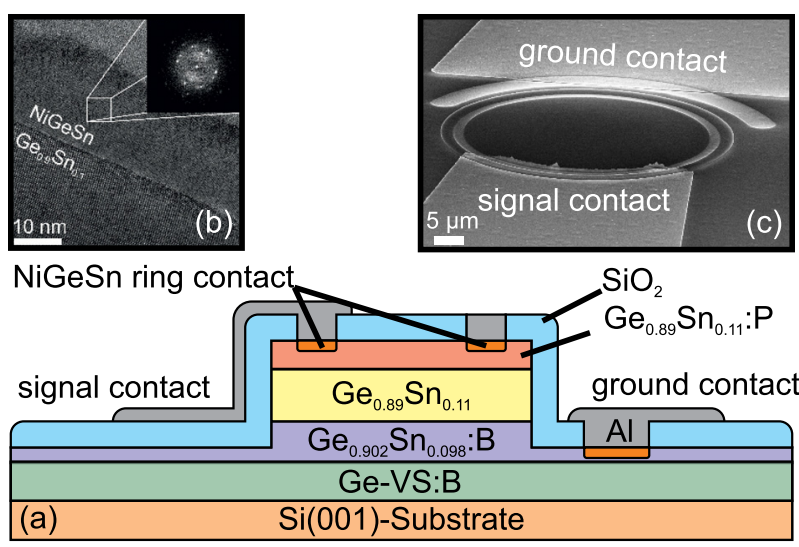

FIG. 2. GeSn $p$ - $i$ - $n$ diode: (a) device sketch; (b) TEM micrograph of NiGeSn on GeSn showing a smooth interface and a well oriented NiGeSn as can be seen by the FFT-inset; and (c) SEM-image of a fabricated $p-i$ - $n$ diode.

GeSn $p$ - $i$ - $n$ diodes were processed by applying standard CMOS technology like reactive ion etching (RIE), optical lithography, and NiGeSn contacts. However, in order to avoid $\mathrm{Sn}$ diffusion, low temperature process modules, below $325^{\circ} \mathrm{C}$, have been developed. A sketch of the device and the layer stack is shown in Fig. 2(a). First, a $250 \mathrm{~nm}$ deep mesa was etched by RIE in order to contact the highly p-doped bottom layer. The following steps were $\mathrm{SiO}_{2}$ passivation by plasma enhanced chemical vapour deposition, contact window opening by RIE and NiGeSn metal contact formation. As can be seen in Fig. 2(b), the NiGeSn forms a smooth semiconductor-metal interface. The formation of the low resistive well oriented $\mathrm{NiGeSn}$ contact has been previously studied $^{18}$ and will not be discussed here any further. The fabrication completes with deposition of $200 \mathrm{~nm}$ thick Al bonding pads. Fig. 2(c) depicts a SEM image of the fabricated device.

Temperature dependent I-V characteristics of a $p-i-n-$ diode with a diameter of $50 \mu \mathrm{m}$ are shown in Fig. 3(a). A distinct region of negative differential resistance is observed for temperatures below $175 \mathrm{~K}$. Three different regimes, typical for Esaki diodes, can be identified: (i) band-to-band tunneling, (ii) excess current due to trap assisted tunneling (TAT), and (iii) drift-diffusion by thermal emission of carriers over the potential barrier. For strong reverse bias, the current only slightly increases with temperature. This is a typical behavior for direct band-to-band tunneling where no phonons are required for momentum conservation. ${ }^{4}$ Here, the bandto-band tunneling rate increases as the bandgap decreases with increasing temperature. The situation is different for slightly positive voltages below the peak-current voltage where BTBT is still dominant, but the band overlap of conduction and valence bands of the $\mathrm{n}$ - and p-type regions is small. Here, the current increases with decreasing temperature which can be explained as follows. The energy

TABLE I. Material parameters of GeSn alloys grown on a Ge-VS under slight tensile strain of $0.15 \%$, obtained by $8 \times 8 \mathrm{k} \cdot \mathrm{p}$ method. The Ge-VS top valence band defines the zero energy. Energies in $(\mathrm{eV})$, masses in $\left(\mathrm{m}_{0}\right) ; 1=[001]$ and $\mathrm{t}=[100]$ direction.

\begin{tabular}{llllllllllllll}
\hline \hline$x_{\mathrm{Sn}}$ & $\epsilon(\%)$ & $E_{\mathrm{HH}}$ & $E_{\mathrm{LH}}$ & $E_{\Gamma}$ & $E_{\mathrm{L}}$ & $m_{\mathrm{LHt}}$ & $m_{\mathrm{LHl}}$ & $m_{\mathrm{HHt}}$ & $m_{\mathrm{HHl}}$ & $m_{\Gamma \mathrm{t}}$ & $m_{\Gamma \mathrm{l}}$ & $m_{\mathrm{Lt}}$ & $m_{\mathrm{Ll}}$ \\
\hline 0.11 & -0.55 & 0.131 & 0.079 & 0.638 & 0.651 & 0.046 & 0.022 & 0.023 & 0.220 & 0.029 & 0.033 & 0.08 & 1.559 \\
0.098 & -0.37 & 0.109 & 0.073 & 0.640 & 0.648 & 0.050 & 0.022 & 0.025 & 0.221 & 0.030 & 0.033 & 0.08 & 1.559 \\
\hline \hline
\end{tabular}



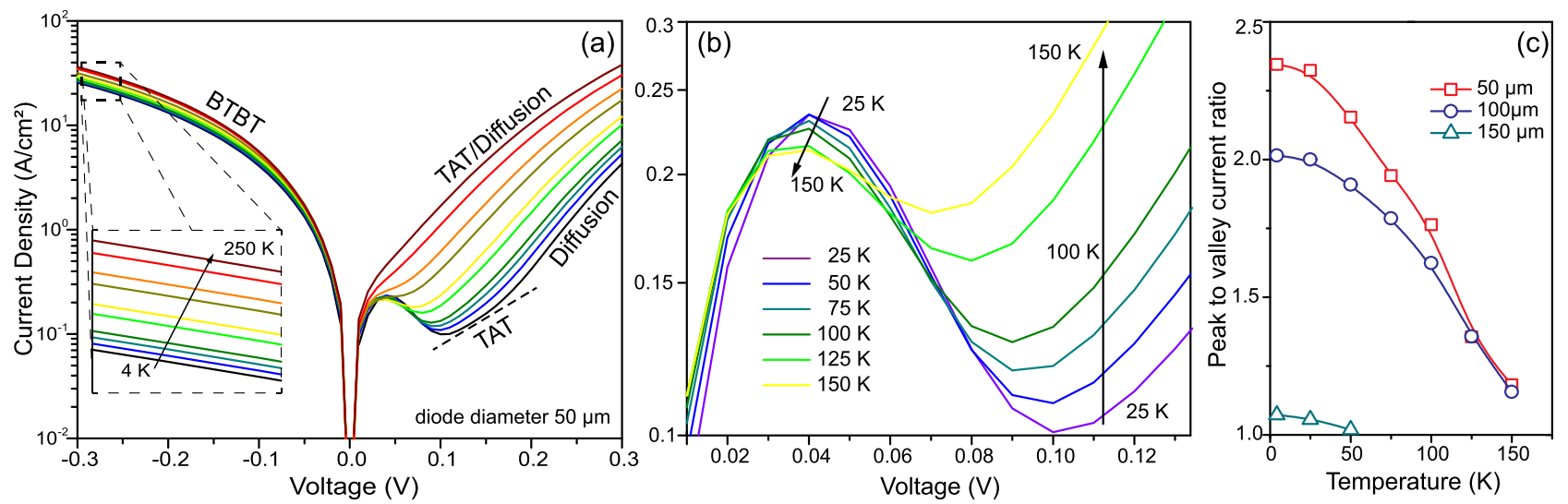

FIG. 3. (a) I-V characteristics of a $p-i-n$ diode with $50 \mu \mathrm{m}$ diameter showing NDR for $T<175 \mathrm{~K}$. (b) Zoom of the NDR region indicating the temperature dependence of the current components. (c) PVCR for different diode sizes plotted against the temperature. The PVCR depends almost linear on the temperature.
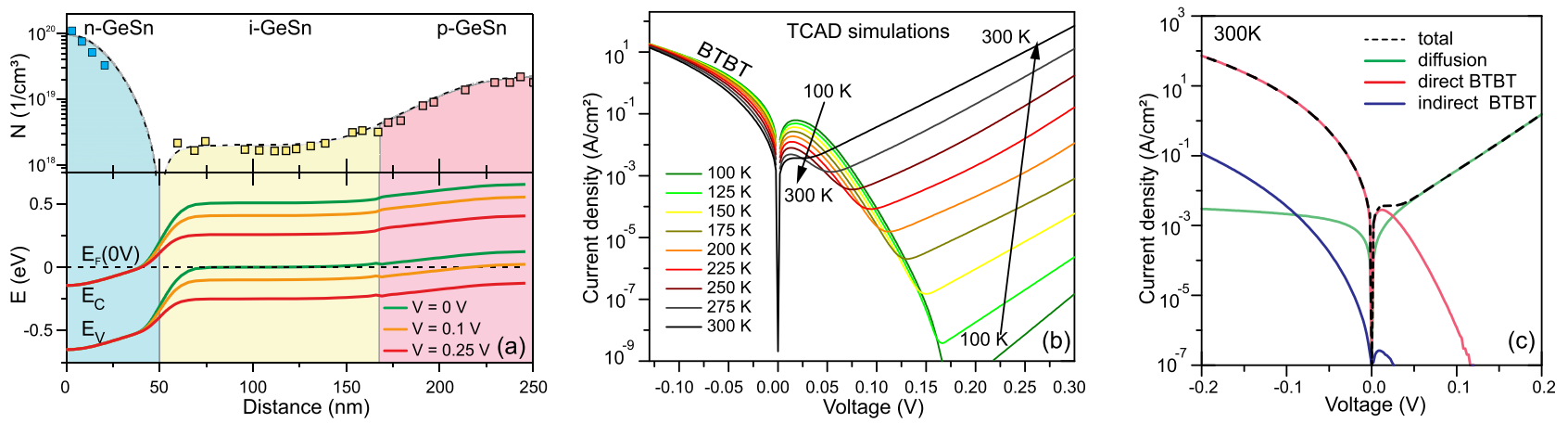

FIG. 4. (a) (Top) Doping profile as obtained by ECV (dots). The fit (dashed line) has been used for the simulation of the electronic band structure alignment of the GeSn $p-i-n$ diode at $T=300 \mathrm{~K}$ (bottom). (b) and (c) Simulated I-V Esaki diode characteristics: (b) temperature dependence and (c) current components at $300 \mathrm{~K}$.

separation of the $\Gamma$ - and L-valleys in the present layer structure is very small, about $13 \mathrm{meV}$. Consequently, both the $\Gamma$ and L-valleys are populated at $300 \mathrm{~K}$ (Fig. 1(d)). By decreasing the temperature, electrons condensate into the $\Gamma$-valley. As the BTBT-probability for direct tunneling is much higher than for indirect tunneling, the increased $\Gamma$-population leads to an increased tunneling current for lower temperatures at low bias (Fig. 3(b)). A further increase of the applied voltage suppresses the BTBT as conduction band of the n-type

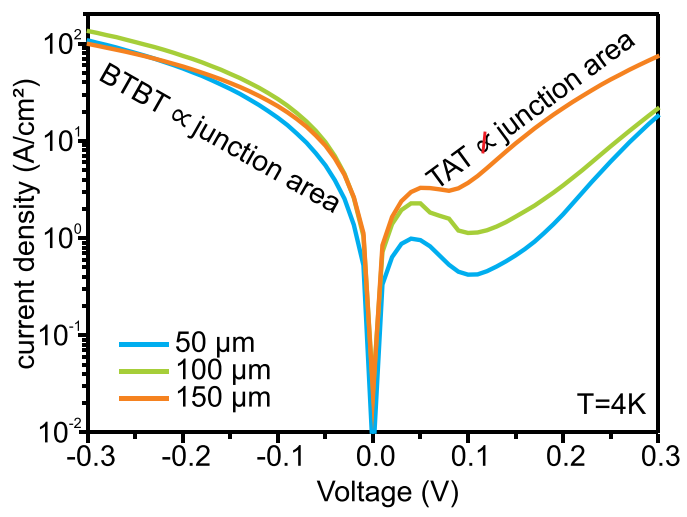

FIG. 5. Esaki diode characteristics for different diode sizes normalized by the area of the top ring electrode. In reverse bias, dominated by BTBT, the I-V curves overlap for different diode sizes as the BTBT-current scales with the junction area. In forward bias, TAT is dominating which does not scale with the junction area. region and valence band of the p-type region do not overlap anymore (Fig. 4(a)) resulting in a decrease of the current and giving rise to a NDR. Peak to valley current ratios (PVCR) of 2.3 and $\sim 2$ have been measured at $4 \mathrm{~K}$ for diodes with $50 \mu \mathrm{m}$ and $100 \mu \mathrm{m}$ diameters, respectively. Above $0.1 \mathrm{~V}$, the BTBT is overlapped by TAT that strongly depends on temperature and causes an excess current. As a consequence, the valley current increases with temperature leading to a typical roughly linear dependence of the PVCR on temperature ${ }^{8}$ (Fig. 3(c)). For larger forward biases, thermal emission over the $p-i-n$ junction barrier comes into play. The low bandgap value of $0.5 \mathrm{eV}$ leads to a strongly enhanced diffusion current. Furthermore, as the (pseudo) intrinsic region is fairly large, the BTBT probability is small in moderate forward bias. Thus, above $175 \mathrm{~K}$ diffusion and TAT currents dominate and NDR vanishes. To analyze the physical mechanisms, simulations using a non-local BTBT model of the TCAD simulator Synopsys Sentaurus Device were performed. The input material parameters are summarized in Table I. The calculated real-space band structure of the $p-i-n$ diode for different applied voltages is shown in Fig. 4(a), indicating clearly a conduction band to valence band overlap of roughly $0.14 \mathrm{eV}$ at zero bias which is crucial for achieving NDR. The simulated Esaki diode characteristics for $300 \mathrm{~K}$ are shown in Fig. 4(c). It should be pointed out that although $\Gamma$ - and L-valleys have nearly the same energy, and the $\mathrm{L}$-valley DOS is larger than for the $\Gamma$-valley, the contribution 
of direct BTBT is $\sim 1000$ times higher than indirect BTBT due to the significant differences in the effective masses and no-phonon involvement. At $300 \mathrm{~K}$, in forward bias, the strong contribution of diffusion current is masking the NDR effect. As the simulation takes into account ShockleyRead-Hall generation/recombination but not TAT, the NDR is visible at higher temperatures than found experimentally. The voltage region of the NDR and the current density values are in good agreement with the experimental data. Further analysis revealed the contributions of BTBT and TAT by comparing different diode sizes. The fabricated $p-i-n$ diodes feature ring contacts instead of classical circular contacts, suitable for light emitting diodes based on the direct bandgap of the $\mathrm{Ge}_{0.89} \mathrm{Sn}_{0.11}$ alloy. The analyses of the $\mathrm{I}-\mathrm{V}$ characteristics for different diode sizes, Fig. 5, indicate as expected that the current in reverse bias scales with the top NiGeSn ring contact area rather than with the circular area of the diode. In reverse bias, BTBT dominates and scales with the junction area. However, in forward bias, TAT dominates which does not scale with the contact area. This is also reflected in Fig. 3(c) showing the PVCR for different temperatures and diode diameters. For larger diodes, the PVCR is smaller and the NDR vanishes at lower temperatures indicating that the contribution of TAT is more pronounced for larger diodes. Point defects are typically incorporated in epitaxial layers grown at lower temperatures, here $350^{\circ} \mathrm{C}$, and presumably the main source of TAT. Optimized GeSn $p-i-n$ structures with a thinner intrinsic region, improved doping profiles, and even lower bandgap are expected to increase the tunneling currents and to reach higher temperature NDR.

In conclusion, we have presented the characterization of GeSn tunneling diodes as a function of temperature. The BTBT current in the direct bandgap $\mathrm{Ge}_{0.89} \mathrm{Sn}_{0.11}$ diodes scales with the contact area. An NDR effect with a PVCR up to 2.3 has been achieved at low temperatures. The appearance of TAT is attributed to point defects which may stem from the ultralow temperature CVD growth. Band-structure and device simulations provided deeper insight in the benefit of direct tunneling even for only slightly direct GeSn alloys due to small effective masses. Further analysis of GeSn tunneling diodes will provide important data such as tunneling masses. The realization of Esaki type GeSn represents an important step towards advanced Si-based tunneling-FETs.

This research received partial funding from the EU FP7 project E2SWITCH (619509) and the BMBF project UltraLowPow (16ES0060 K).

${ }^{1}$ S. Wirths, R. Geiger, N. von den Driesch, G. Mussler, T. Stoica, S. Mantl, Z. Ikonic, M. Luysberg, S. Chiussi, J. M. Hartmann, H. Sigg, J. Faist, D. Buca, and D. Grützmacher, Nat. Photonics 9, 88 (2015).

${ }^{2}$ Y. Yang, G. Han, P. Guo, W. Wang, X. Gong, L. Wang, K. L. Low, and Y.-C. Yeo, IEEE Trans. Electron Devices 60, 4048 (2013).

${ }^{3}$ S. Wirths, A. T. Tiedemann, Z. Ikonic, P. Harrison, B. Holländer, T. Stoica, G. Mussler, M. Myronov, J. M. Hartmann, D. Grützmacher, D. Buca, and S. Mantl, Appl. Phys. Lett. 102, 192103 (2013).

${ }^{4}$ Q. Smets, D. Verreck, A. S. Verhulst, R. Rooyackers, C. Merckling, M. Van De Put, E. Simoen, W. Vandervorst, N. Collaert, V. Y. Thean, B. Sorée, G. Groeseneken, and M. M. Heyns, J. Appl. Phys. 115, 184503 (2014).

${ }^{5}$ L. Esaki, Phys. Rev. 109, 603 (1958).

${ }^{6}$ M. Oehme, Thin Solid Films 520, 3341 (2012).

${ }^{7}$ M. Oehme, A. Karmous, M. Sarlija, J. Werner, E. Kasper, and J. Schulze, Appl. Phys. Lett. 97, 012101 (2010).

${ }^{8}$ M. Stoffel, G. S. Kar, and O. G. Schmidt, Mater. Sci. Eng., C 25, 826 (2005).

${ }^{9}$ M. T. Björk, H. Schmid, C. D. Bessire, K. E. Moselund, H. Ghoneim, S. Karg, E. Lörtscher, and H. Riel, Appl. Phys. Lett. 97, 163501 (2010).

${ }^{10}$ S. Gupta, B. Vincent, B. Yang, D. Lin, F. Gencarelli, J.-Y. J. Lin, R. Chen, O. Richard, H. Bender, B. Magyari-Köpe, M. Caymax, J. Dekoster, Y. Nishi, and K. C. Saraswat, in IEEE International Electron Devices Meeting (IEDM) (IEEE, 2012), pp. 16-2.

${ }^{11}$ G. Han, S. Su, C. Zhan, Q. Zhou, Y. Yang, L. Wang, P. Guo, W. Wei, C. P. Wong, Z. X. Shen, B. Cheng, and Y.-C. Yeo, in IEEE International Electron Devices Meeting (IEDM) (IEEE, 2011), pp. 16-7.

${ }^{12}$ Y. Yang, K. Lu Low, W. Wang, P. Guo, L. Wang, G. Han, and Y.-C. Yeo, J. Appl. Phys. 113, 194507 (2013).

${ }^{13}$ S. Gupta, B. Magyari-Köpe, Y. Nishi, and K. C. Saraswat, J. Appl. Phys. 113, 073707 (2013).

${ }^{14}$ T. B. Bahder, Phys. Rev. B 41, 11992 (1990).

${ }^{15}$ K. Lu Low, Y. Yang, G. Han, W. Fan, and Y.-C. Yeo, J. Appl. Phys. 112, 103715 (2012).

${ }^{16}$ S. Wirths, Z. Ikonic, A. T. Tiedemann, B. Holländer, T. Stoica, G. Mussler, U. Breuer, J. M. Hartmann, A. Benedetti, S. Chiussi, D. Grützmacher, S. Mantl, and D. Buca, Appl. Phys. Lett. 103, 192110 (2013).

${ }^{17}$ Y. Bogumilowicz and J. M. Hartmann, Thin Solid Films 557, 4 (2014).

${ }^{18}$ S. Wirths, R. Troitsch, G. Mussler, J.-M. Hartmann, P. Zaumseil, T. Schroeder, S. Mantl, and D. Buca, Semicond. Sci. Technol. 30, 055003 (2015). 